\title{
Erratum:
}

\section{Erratum to: Fast global kernel fuzzy c-means clustering algorithm for consonant/vowel segmentation of speech signal}

\author{
Xian ZANG ${ }^{1}$, Felipe P. VISTA IV ${ }^{1}$, Kil To CHONG $^{1,2}$ \\ ( ${ }^{1}$ Department of Electronic Engineering, Jeonbuk National University, Jeonju-si 561-756, Korea) \\ ( ${ }^{2}$ Advanced Electronics and Information Research Center, Jeonbuk National University, Jeonju-si 561-756, Korea) \\ E-mail: zangxian@jbnu.ac.kr; boduke@jbnu.ac.kr; kitchong@jbnu.ac.kr
}

doi: $10.1631 /$ jzus.C13e0320

Erratum to: J Zhejiang Univ-Sci C (Comput \& Electron) 2014 15(7):551-563 doi:10.1631/jzus.C1300320

The original version of this article unfortunately contained mistakes. Algorithm 6 should be as follows:

\section{Algorithm 6 FGKFCM-F clustering} Input:

(1) $X=\left\{\boldsymbol{x}_{1}, \boldsymbol{x}_{2}, \ldots, \boldsymbol{x}_{N}\right\}, \boldsymbol{x}_{i} \in \mathbb{R}^{d}, i=1,2, \ldots, N$, the dataset;

(2) $C, 1<C \leq N$, the number of clusters;

(3) $\varepsilon>0$, the stopping criterion;

(4) $\boldsymbol{\mu}^{(0)}=\left(\boldsymbol{\mu}_{1}^{(0)}, \boldsymbol{\mu}_{2}^{(0)}, \ldots, \boldsymbol{\mu}_{C}^{(0)}\right)$, the initials of memberships;

(5) $m>1$, the weighting exponent;

(6) $\sigma$, the GRBF kernel parameter.

Output:
(1) $\tilde{\boldsymbol{v}}=\left(\tilde{\boldsymbol{v}}_{1}, \tilde{\boldsymbol{v}}_{2}, \ldots, \tilde{\boldsymbol{v}}_{C}\right)$, the final cluster prototypes;
(2) $\boldsymbol{\mu}=\left(\boldsymbol{\mu}_{1}, \boldsymbol{\mu}_{2}, \ldots, \boldsymbol{\mu}_{C}\right)$, the final memberships.

1 Compute $\tilde{\boldsymbol{v}}^{*}(1)$ using Eq. (23) with initial position $\tilde{\boldsymbol{v}}(1)$ placed at the data point that minimizes Eq. (24);

2 for $k=2$ to $C$ do

3 for $n=1$ to $N$ do
4

Set the initial state

$$
\begin{aligned}
\tilde{\boldsymbol{v}}^{n(0)}(k) & =\left(\tilde{\boldsymbol{v}}_{1}(k), \tilde{\boldsymbol{v}}_{2}(k), \ldots, \tilde{\boldsymbol{v}}_{k-1}(k), \tilde{\boldsymbol{v}}_{k}(k)\right) \\
& =\left(\tilde{\boldsymbol{v}}_{1}^{*}(k-1), \tilde{\boldsymbol{v}}_{2}^{*}(k-1), \ldots, \tilde{\boldsymbol{v}}_{k-1}^{*}(k-1), \boldsymbol{x}_{n}\right) ;
\end{aligned}
$$

5

Set initial memberships $\boldsymbol{\mu}^{n(0)}(k)$ with respect to

$\tilde{\boldsymbol{v}}^{n(0)}(k)$ using Eq. (25);

6 end

$7 \quad l=\arg \min J^{\Phi}\left(\boldsymbol{\mu}^{n}(k)\right)$ using Eq. (19);

$8 \quad \boldsymbol{\mu}^{n}(k) \leftarrow \operatorname{KFCM}-\mathrm{F}\left(X, k, \varepsilon, m, \sigma^{2}, \boldsymbol{\mu}^{n(0)}(k)\right)$;

$9 \quad \tilde{\boldsymbol{v}}^{(0)}(k) \leftarrow \tilde{\boldsymbol{v}}^{l(0)}(k)$;

$10 s=1$;

11 Update $\tilde{\boldsymbol{v}}^{(s)}(k)$ with $\tilde{\boldsymbol{v}}^{(s-1)}(k)$ using Eq. (23);

12 If $\left\|\tilde{\boldsymbol{v}}^{(s)}(k)-\tilde{\boldsymbol{v}}^{(s-1)}(k)\right\|<\varepsilon$

13 STOP and $\tilde{\boldsymbol{v}}^{*}(k) \leftarrow \tilde{\boldsymbol{v}}^{(s)}(k)$;

14 Else $s=s+1$ and return to line 11;

15 end

$16 \tilde{\boldsymbol{v}} \leftarrow \tilde{\boldsymbol{v}}^{*}(C), \boldsymbol{\mu} \leftarrow \boldsymbol{\mu}^{*}(C)$. 